\title{
Coexistence Performance and Limits of Frame-Based Listen-Before-Talk
}

\author{
Gordon J. Sutton, Ren Ping Liu, Senior Member, IEEE, and Y. Jay Guo, Fellow, IEEE
}

\begin{abstract}
Frame-based listen-before-talk (FB-LBT) has been adopted as one of the channel access mechanism for Wi-Fi/LTE coexistence. We aim to explore the limits of FB-LBT by developing theoretical models to characterise the FB-LBT channel access performance under the coexistence of LTE and Wi-Fi. We first derive a steady-state model to calculate the spectrum share occupied by LTE under the assumption that the Wi-Fi transmissions have stationary distributions. The assumption does not hold when the time between two LTE transmissions is short, where the system is dominated by a dynamic phenomenon. A second model is developed that accounts for the dynamics of the Wi-Fi channel access mechanism. Our models, validated by simulation results, accurately calculate the spectrum share occupied by LTE over a range of FB-LBT frame periods and Wi-Fi traffic loads. We obtain upper bounds on the FB-LBT spectrum share when competing with heavy Wi-Fi traffic, which confirm the weakness of FB-LBT. Moreover, we demonstrate that our models can be used to control the FB-LBT spectrum share within a modest range.
\end{abstract}

Index Terms-Frame-based equipment, LTE-Wi-Fi coexistence, listen-before-talk, Fairness.

\section{INTRODUCTION}

The scarcity of licensed spectrum for cellular communications below $6 \mathrm{GHz}$ has motivated the consideration of using the unlicensed bands to suppliment the operation of LTE. However, the usage of LTE in unlicensed spectrum creates numerous challenges since LTE physical channels have largely been designed on the basis of uninterrupted operation on licensed carriers. In contrast, the unlicensed spectrum is traditionally the domain of $\mathrm{Wi}-\mathrm{Fi}$, which operates under the long standing, yet evolving, IEEE 802.11 protocol [1], [2], where channel access is unscheduled and based on contention. As such, one challenge for LTE in the unlicensed spectrum is to coexist with Wi-Fi in a fair manner, such that both technologies can obtain a fair portion of the channel time.

To help facilitate fair channel sharing, Listen-Before-Talk (LBT) has been introduced [3], [4]. In LBT, the eNB performs a clear channel assessment (CCA) to assess the channel for transmissions from other users prior to commencing its own transmission. If the channel is sensed busy, the eNB does not transmit, and instead repeats the channel assessment process at a later time. The LBT process both reduces the number of collisions and allows other devices a chance to access the channel.

The throughput performance of a simple LBT scheme was analysed in [5] via Markov chain models. Their work

G.J. Sutton, R.P. Liu, and Y.J. Guo are with the Global Big Data Technologies Centre, University of Technology Sydney, Australia (e-mail: firstname.lastname@uts.edu.au). confirmed the effectiveness of LBT in Wi-Fi/LTE coexistence scenarios. LBT transmit power control and channel sensing threshold were studied by simulations in [6], [7]. A channelsensing based access scheme operating at the subframe level was proposed and analysed in [8]. An enhanced LBT scheme was proposed and analysed in [9] by simulations. The above simulation and analysis results show that LBT is effective for enabling Wi-Fi/LTE coexistence even in dense deployments.

In the European Telecommunications Standards Institute (ETSI) standard [10], LBT operation procedures were categorized as being either frame-based LBT (FB-LBT) or loadbased LBT (LB-LBT), where FB-LBT has a fixed timing structure for transmit-receive and LB-LBT has a dynamic load-based timing structure for transmit-receive. FB-LBT has the advantage that it aligns more readily with the LTE subframe/frame structure. However, it is unclear that FB-LBT, as given in [10], can compete with high Wi-Fi loads, and there has not been quantitative analysis on the performance or bounds of its channel access.

Wi-Fi and LTE LBT protocols rely on both sensing the channel and a set of timing rules to determine whether to transmit and what to do if there is a collision. MAC-layer coexistence models have been developed that incorporate the effect of protocol timings and collisions on channel access, while assuming the channel sensing is accurate. PHY-layer models have also been developed that focus on the channel sensing and SINR, while paying less attention to the detailed protocol timings. The MAC-layer models are appropriate when the devices are all within a shared coverage area, such as in indoor environment. The PHY-layer models are more appropriate when the distances between devices are large, such as in outdoor environments, where high attenuation can lead to transmissions not always being detected and a degree of spatial re-use.

Using the MAC-layer approach, a FB-LBT variant was analysed in [11], in which the eNB transmits a dummy packet prior to the each CCA to block Wi-Fi access and reserve the channel. However, this reservation process is not in keeping with the ETSI FB-LBT, and the Wi-Fi access protocol is modelled as a 1-persistent process, rather than the standard exponential backoff process. There have been analyses of a number of LB-LBT schemes based on stationary Markov models, including [12]-[18].

Coexistence models that focus on PHY-layer aspects include [19] and [20]. In [19], a homogeneous point Poisson processes framework is used to spatially model interference between network nodes under a variety of LTE coexistence access mechanisms, including unmodified LTE, LTE-U, and LB-LBT. 
The complicated Wi-Fi backoff process timing is simplified to a continuous variable, uniformly distributed on $[0,1]$, which defines a channel access order, while keeping the model tractable. In [20], distances between devices are modelled by a spiral approximation within a continuum field, with rings around devices representing the limits of carrier sensing and energy detection. However, the model is designed for an outdoor setting and there is no model component to account for the timings of the Wi-Fi and LBT channel access processes.

These aforementioned publications do not model the FBLBT of the ETSI standard [10], nor do they explore any dynamic interactions with Wi-Fi under high Wi-Fi load.

In this paper, we develop theoretical models to characterise the channel access performance of the FB-LBT scheme prescribed in the ETSI standard [10] when applied to indoor Wi-Fi/LTE coexistence scenarios. In particular, our aim is to determine the performance limits between an eNB competing with a number of Wi-Fi STAs, or backoff entities. To this end, we consider saturated LTE and Wi-Fi traffic, on the premise that if any device's load were to lighten, the other devices in the system would be no worse off. An initial steadystate model is developed under the assumption that the Wi-Fi traffic has sufficient time to reach stationarity before each LTE clear channel assessment. The steady-state model is validated when the idle period between LTE transmissions is much longer than the Wi-Fi packet transmission time. However, when the idle period is short, i.e., comparable with Wi-Fi packet transmission time, a dynamic phenomenon causes the spectrum share to deviate from the steady-state model.

We then develop a dynamic model to account for the dynamic evolution of the Wi-Fi state distributions within a short idle period. Our model, validated by simulations, accurately calculates the spectrum share occupied by LTE under a range of FB-LBT frame periods and Wi-Fi traffic loads. The dynamic model reveals that the spectrum share has a damped-oscillatory pattern, as a function of the FB-LBT frame period. For longer frame periods, the oscillations subside and the steady-state model provides a good approximation to the spectrum share.

We obtain the upper bounds of the spectrum share for FBLBT over a range of Wi-Fi technologies. Our results confirm the hypothesis that FB-LBT is unable to attain a fair time share of the unlicensed spectrum, reaching a maximum of $32 \%$, under the coexistence with common Wi-Fi technology, such as IEEE $802.11 \mathrm{n}(20 \mathrm{MHz})$, and reaching a maximum of $46 \%$ channel share, under the coexistence with advanced WiFi technology, i.e. IEEE $802.11 \mathrm{ac}(160 \mathrm{MHz})$. Moreover, our model reveals that the spectrum share can be controlled within a modest range by altering the FB-LBT frame period.

The rest of the paper is organised as follows. Section II introduces FB-LBT and its coexistence operation with Wi-Fi, and gives assumptions. Section III develops the steady-state model and the dynamic model that are used to characterise FB-LBT coexistence performance. Section IV validates the models, explores the performance of FB-LBT, obtains bounds on spectrum share, and discusses control mechanisms. Concluding remarks are given in Section V.

\section{Coexistence Scenario}

We first describe Frame-Based Listen-Before-Talk, and then describe its coexistence operation with Wi-Fi.

\section{A. Frame-Based Listen-Before-Talk}

In Frame-Based Listen-Before-Talk (FB-LBT) [10], a clear channel assessment (CCA) is performed periodically at predefined time instances according to a predetermined frame structure, with periodicity of Fixed Frame Period $T_{F F P}$, as shown in Fig. 1. The Channel Occupancy Time $T_{L T E}$ is designated for transmissions of up to 10 LTE subframes, and shall not exceed $10 \mathrm{~ms}$. The Idle Period $T_{\text {Idle }}$ is left for access by other technologies, e.g., Wi-Fi hot spots. The minimum Idle Period shall be at least $5 \%$ of the Channel Occupancy Time [10]. If the equipment finds the Operating Channel(s) to be clear for the entire duration of a CCA, $T_{C C A}$, it may transmit immediately for $T_{L T E}$. If the equipment finds an Operating Channel occupied, it shall not transmit on that channel during the following Fixed Frame Period, leaving $T_{F F P}$ for Wi-Fi transmissions.

\section{B. FB-LBT Coexistence with Wi-Fi}

The Wi-Fi devices access the channel using the IEEE 802.11 DCF [1], which is a slot-based exponential backoff process, also known as carrier-sense multiple access with collision avoidance (CSMA/CA).

The slots are either short, when the channel is sensed idle, or the duration of a transmission exchange, when the channel is sensed busy, ending after the channel is sensed idle for a continuous distributed interframe space (DIFS). The WiFi transmissions are taken to have duration $T_{W i F i}$, which includes an initial short transition time $\delta$ and a concluding DIFS of silence, as shown in Fig. 1. The transition time $\delta$ includes $\mathrm{Rx}$ to Tx turnaround time and radio propagation time. The LTE frames have a duration $T_{L T E} \gg T_{W i F i}$, and also include an initial transition time $\delta$ and a concluding DIFS of silence, as shown in Fig. 1.

When a Wi-Fi station (STA) is in the idle state and has a new packet to transmit, it first performs carrier sensing to determine the channel activity. If the channel is idle for a DIFS, the STA transmits. Otherwise, the STA begins a backoff procedure comprising a sequence of backoff stages, each ending with a transmission, that continue until either a transmission is successful or a maximum retry limit is reached. The initial backoff stage is backoff stage- 0 , the next is backoff stage-1, etc.

In backoff stage-0, an initial integer backoff counter is selected from the contention window $\left[0, . ., W_{0}-1\right]$ with uniform distribution. The backoff counter is then decremented each time the channel is sensed idle for a slot time $\sigma$. When the channel is instead sensed busy, the countdown is 'frozen' until the channel is sensed idle for a continuous DIFS, and then the countdown continues. When the backoff counter reaches zero, the STA transmits.

If the transmission is unsuccessful, the next backoff stage commences. Subsequent backoff stages follow the same process, but with longer contention windows. $W_{i}$ doubles each 


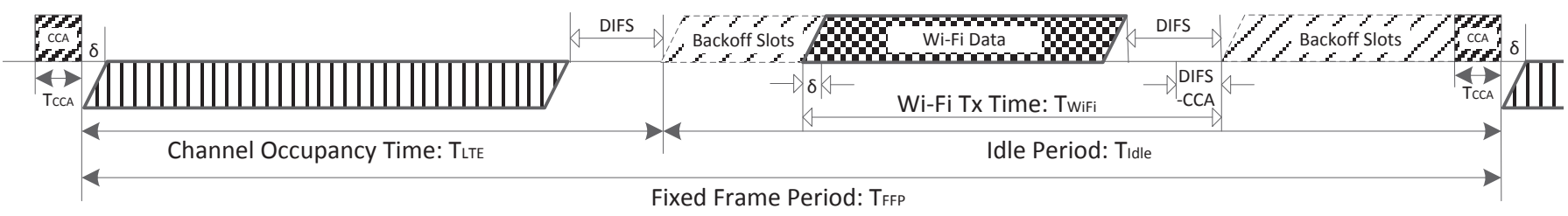

Fig. 1. LBT Frame Structure and Timing

backoff stage up to a maximum size $W_{m}$ and then remains at $W_{m}$ until the maximum backoff stage, $s$, so that $W_{i}=$ $2^{\min (i, m)} W_{0}$, where $i=0, . ., s$.

Once a transmission is successful, or the retransmission limit is reached, the STA waits a DIFS, and then begins a new backoff stage- 0 process. If the backoff counter then reaches zero again and the STA has no packet to transmit, the STA returns to the idle state.

In the coexistence scenario, a busy slot might be due to a Wi-Fi transmission from a STA, or an LTE frame transmission. The right end of Fig. 1 demonstrates an LTE frame interrupting the Wi-Fi STAs' backoff countdown processes. The CCA finds the channel clear, so the eNB begins an LTE frame transmission and, after $\delta$, the transmission is audible to the rest of the network, so the STAs freeze their backoff countdown processes. The middle of Fig. 1 demonstrates the resumption of the Wi-Fi STAs' backoff countdown processes after an LTE transmission finishes and the channel has been idle for a DIFS. Once a STA's backoff counter reaches zero, it transmits and, after $\delta$, the transmission is audible to the rest of the network, so the other STAs freeze their backoff countdown processes. After the Wi-Fi completes its transmission and the channel is idle for a DIFS, the Wi-Fi STAs' backoff countdown processes resume again.

The Wi-Fi slots are synchronized such that when multiple STAs have the same backoff counter, they will count down to zero simultaneously, and their transmissions will collide. The LTE frames are not synchronized with the Wi-Fi slots. Although an LTE CCA senses Wi-Fi transmissions, if the CCA finishes within $\delta$ of when a Wi-Fi transmission would commence, the channel will appear clear to both Wi-Fi and LTE at their respective transmission-decision points, so both the Wi-Fi and LTE transmissions will proceed, and then subsequently collide. The Wi-Fi header conveys control information at the start of each Wi-Fi transmission, whereas LTE control information is transmitted at the start of each LTE subframe for the subframe [21], [22]. As such, Wi-Fi packets are lost in a collision, whereas only the LTE subframes involved in the collision are lost, which is further discussed in Section III-C

\section{FB-LBT COEXISTENCE Models}

We consider a Wi-Fi/LTE coexistence scenario comprising an LTE base station (eNB) supporting a number of user equipment (UE) and $N$ Wi-Fi stations (STAs). The eNB accesses the unlicensed spectrum using frame-based Listen-Before-Talk (FB-LBT), and once successful, transmits a downlink LTE data frame to the $\mathrm{UE}^{1}$. The Wi-Fi STAs access the channel using the 802.11 DCF protocol and are assumed to have saturated traffic.

We aim to characterise the FB-LBT Wi-Fi/LTE coexistence performance. In particular, we derive the time-average probability of the CCA finding the channel 'clear', from which the channel share and throughput can be obtained. An initial steady-state model is developed based on the assumption that, at the time of each CCA, the probability distribution of the Wi-Fi STAs being in the various states of the CSMA/CA process equals the stationary steady-state distribution for $N$ saturated STAs. Such an assumption is valid when the idle period is long. However, when the Idle period is short $(\lesssim 4$ $\mathrm{ms}$ ), a dynamic phenomenon is observed in simulations. A second model is then developed that accounts for the dynamic evolution of the Wi-Fi state distribution during short Idle Periods.

\section{A. Steady-state Model}

The coexistence performance of the eNB can be charactersied by how often the eNB gains access to the channel. The eNB performs a Clear Channel Assessment (CCA) once per Fixed Frame Period (FFP), just before the FFP starts, as shown in Fig. 1. If the CCA finds the channel 'clear' the eNB immediately transmits an LTE-block; otherwise, the CCA finds the channel 'busy' and the eNB does not transmit during the following FFP. Denote the long-term probability that a CCA finds the channel clear $P_{C C}$, and denote the proportion of time the channel is occupied by the eNB $\rho_{L T E}$. Then $\rho_{L T E}$ can be estimated as

$$
\rho_{L T E}=P_{C C} \frac{T_{L T E}}{T_{F F P}},
$$

where $T_{L T E}$ is the duration of an LTE-block transmission and $T_{F F P}$ is the duration of a FFP.

During the LTE-block transmissions, the Wi-Fi STAs sense the channel as 'busy' and 'freeze' their access processes. When $T_{I d l e}$ is relatively long compared to the Wi-Fi packet transmission times, the behaviour of the Wi-Fi STAs can be approximately modelled by a network comprising just the $N$ saturated Wi-Fi STAs, with the LTE-block transmissions reducing the $\mathrm{Wi}-\mathrm{Fi}$ access time, but otherwise having little impact on the Wi-Fi transmission processes.

A model is developed in this section for $P_{C C}$ under the assumption that the $N$-STAs have reached their steady-state distribution by the time of each CCA. The model effectively

\footnotetext{
${ }^{1}$ Only downlink traffic is transmitted in unlicensed spectrum, uplink traffic is transmitted through licensed bands [3].
} 
decouples the eNB from the Wi-Fi STAs. When the LTE transmissions are relatively infrequent, the Wi-Fi part of the system has time to approach its (without LTE traffic) steadystate distribution and this decoupling approximation has little impact on the model's accuracy. To estimate $P_{C C}$ under this assumption, a reduced network comprising just the $N$ STAs is modelled as per [23], which is based on [24], where the DCF mechanism for a single STA is modelled using an embedded Markov chain, with states specified by the 2-tuple: (backoff stage, backoff countdown) that transition at the start of each MAC slot.

The Markov chain inputs the collision probability, given the STA is transmitting, which is denoted $p$. The probability that the STA transmits during each MAC slot, denoted $\tau$, is extracted from the steady-state distribution, and can be calculated as

$$
\tau=\frac{2\left(1-p^{s+1}\right)}{(1-p) \sum_{i=0}^{s}\left(W_{i}+1\right) p^{i}} .
$$

A network interaction equation also relates $\tau$ and $p$, for a specified $N$ and based on the approximation that the STAs' DCF processes are independent, as

$$
p=1-(1-\tau)^{N-1} \text {. }
$$

$\tau$ and $p$ are then obtained by simultaneously solving (2) and (3). Denote the marginal probability that no STA transmits during a given MAC slot $P_{\text {noTx }}$. Then

$$
P_{\text {noTx }}=(1-\tau)^{N},
$$

and the average duration of a MAC slot, denoted $E_{s}$, is

$$
E_{s}=P_{\text {noTx }} \sigma+\left(1-P_{\text {noTx }}\right) T_{W i F i},
$$

where $T_{W i F i}$ is the expected duration of a MAC slot in which at least one STA is transmitting.

(2)-(5) form the stationary Wi-Fi model from which $P_{C C}$ can be estimated. However, $P_{C C}$ cannot be obtained directly from the above Markov model because Frame-based LBT has different slot timings and contention mechanisms to the $\mathrm{Wi}-\mathrm{Fi}$ STAs. In particular, the CCA could end anytime during either a transmission or non-transmission MAC slot. $P_{C C}$ is estimated as the expected value, calculated over the time probabilities of each type of MAC slot occurring, of a CCA being found 'clear' when the CCA ends during the type of MAC slot.

At the start of any MAC slot, the channel will have been 'clear' for at least a DIFS. Hence, a CCA will find the channel 'clear' when the CCA ends during:

- any of the non-transmission backoff MAC slots;

- the final $D I F S-T_{C C A}$ of a transmission MAC slot; or

- the initial $\delta$ of a transmission MAC slot, while a Wi-Fi STA is transitioning from $\mathrm{Rx}$ to Tx operation.

These three CCA-timing categories have respective durations of: $\sigma, D I F S-T_{C C A}$ and $\delta$, giving $P_{C C}$ as:

$$
P_{C C}=\frac{P_{\mathrm{noTx}} \sigma+\left(1-P_{\mathrm{noTx}}\right)\left(D I F S-T_{C C A}+\delta\right)}{E_{s}} .
$$

As mentioned in Section II-B, if the CCA finishes within $\delta$ of the start of a Wi-Fi transmission, the channel will be found 'clear', so an LTE frame will be transmitted and the Wi-Fi and
LTE transmissions will collide. The probability of a given LTE transmission colliding with a Wi-Fi transmission, denoted $p_{L}$, is then

$$
p_{L}=2 \delta\left(1-P_{\mathrm{noTx}}\right) /\left(E_{s} P_{C C}\right)
$$

\section{B. Dynamic model}

The model in Section III-A is valid when there is enough time between LTE transmissions for the Wi-Fi STAs to approach their stationary steady-state distribution. Otherwise, the time-varying Wi-Fi state distributions alter $P_{C C}$. In this section we develop a dynamic model to estimate the shortterm variations in the probability of a 'clear' CCA, which are then combined to estimate $P_{C C}$.

The dynamic model follows the evolution of the Wi-Fi STAs' state distributions between LTE transmissions. Take the end of each LTE transmission as time zero. The WiFi STAs have synchronised MAC slots of duration either $\sigma$ or $T_{W i F i}$, so the MAC slots are restricted to commence at integer combinations of $\sigma$ and $T_{W i F i}$. The FB-LBT CCAs occur at the set times $T_{\text {Idle }}+a T_{F F P}$, for $a \in\{0,1, .$.$\} , where$ $T_{\text {Idle }}=T_{F F P}-T_{L T E}$ is the duration of the Idle Period. That is, after an LTE transmission, the first CCA finishes exactly one Idle Period later, so, when the Idle Period is relatively short, this restricted MAC-slot timing affects $P_{C C}$. If the first CCA finds the channel busy, the eNB waits a further $T_{F F P}-T_{C C A}$ before its next CCA, by which time the restricted MAC-slot timing structure has much less influence.

Let $P_{C C}(r)$ be the probability that it is on the $r^{t h} \mathrm{CCA}$ after an LTE transmission that the channel is found 'clear', whereupon the eNB transmits an LTE block. The average number of FFPs required for the system to return to an initial point of 'just after an LTE transmission', i.e. the average return length in FFPs, is denoted $A R L$. As more FFPs pass, the state distribution mixes more over time and approaches the steadystate distribution for $N$ saturated STAs. As such, $P_{C C}(r)$, and in turn $A R L$, can be approximated from the first $R$ values of $P_{C C}(r)$, using geometric and differentiated-geometric series. $P_{C C}$ is then the reciprocal of $A R L$. That is

$$
\begin{aligned}
A R L= & \sum_{a=1}^{\infty} a P_{C C}(a) \\
\approx & \sum_{a=1}^{R} a P_{C C}(a)+ \\
& \beta\left(\frac{1}{(1-\beta)^{2}}+\frac{R}{1-\beta}\right) P_{C C}(R), \\
P_{C C}= & 1 / A R L,
\end{aligned}
$$

where $\beta$ is an estimate of the ratio of consecutive $P_{C C}(r)$ 's (i.e., the probability of a successful CCA, conditional on commencing the CCA) for return lengths $r>R$. For example,

$$
\beta=P_{C C}(R) / P_{C C}(R-1) .
$$

An alternative estimate of $\beta$ is obtained by averaging the last $b$ ratios of consecutive $P_{C C}(r)$ 's, giving

$$
\beta=\frac{1}{b} \sum_{a=R-b+1}^{R} P_{C C}(a) / P_{C C}(a-1) .
$$


Setting $b$ to 9 attempts to account for small variations in $P_{C C}(r)$ associated with the timing of consecutive nontransmission MAC slots through the CCA window, each of duration $9 \mu \mathrm{s}$.

To evaluate $P_{C C}(r)$, the state distribution of a representative STA is propagated, on a micro-second scale, from just after an LTE transmission concludes until just after the next LTE transmission concludes, which may occur after one CCA, or many.

Let $k$ be a count of the micro seconds after an LTE transmission concludes. Since the LTE transmission concludes with a final DIFS of silence, the first MAC slot commences at the start of count $k=1$. Also, let previously defined times, when augmented with an additional $u$ superscript, be duration counts in number of micro seconds, e.g., $\sigma^{u}, T_{W i F i}^{u}, T_{C C A}^{u}$ and $D I F S^{u}$.

The state distributions are recorded in vectors of conditional probabilities. Let $S_{k}$ be a vector of length $\left(2^{s}-1\right) W_{0}$ representing the probability of a representative saturated STA being in the different states of the $\mathrm{Wi}-\mathrm{Fi}$ access process at time $k$, given the STA commences a MAC slot at time $k$. The first $W_{0}$ elements of $S_{k}$, denoted $S_{k}[1], . ., S_{k}\left[W_{0}\right]$ are the probabilities of being in backoff stage- 0 with backoff counts $0, . ., W_{0}-1$. The next $2 W_{0}$ elements, $S_{k}\left[W_{0}+1\right], . ., S_{k}\left[3 W_{0}\right]$ are the probabilities of being in backoff stage- 1 with backoff counts $0, \ldots, 2 W_{0}-1$. And so on, with the final $2^{m} W_{0}$ elements, $S_{k}\left[\left(2^{s}-1-2^{m}\right) W_{0}+1\right], . ., S_{k}\left[\left(2^{s}-1\right) W_{0}\right]$ being the probabilities of being in backoff stage- $s$ with backoff count $0, \ldots, 2^{m} W_{0}-1$.

Further, let $b_{i}^{0}$ and $b_{i}^{f}$, denote the indices of $S_{k}$ corresponding respectively to states with backoff count 0 and backoff count $W_{i}-1$ for backoff stage- $i, i=0, . ., s$, such that

$$
\begin{aligned}
b_{i}^{0} & =\left(2^{i}-1\right) W_{0}+1, \\
b_{i}^{f} & =\left(2^{i+1}+1\right) W_{0} ;
\end{aligned}
$$

and let $b^{f}$ be the set $\left\{b_{0}^{f}, \ldots, b_{s+1}^{f}\right\}$.

All the Wi-Fi STAs have synchronised MAC slots, but the eNB is not synchronised with the Wi-Fi MAC slots. The transition delay, $\delta$, occuring before transmissions, causes a $2 \delta$ window centred about the end of the CCA, during which the commencement of a Wi-Fi transmission MAC slot will cause an LTE/Wi-Fi collision. To create a $2 \delta$ window when using discrete time increments, an ordering of LTE and WiFi transmissions is used, to avoid the window being a micro second too wide. To this end, all transmissions are considered to commence at the 'start' of the micro-second count, however, the LTE transmissions are modelled as occurring just before the Wi-Fi transmissions. All transmissions are modelled as being instantly received after $\delta$.

1) Propagating the representative distribution $S_{k}$ : Let $m_{k}$ be the probability of a MAC slot commencing at time $k . m_{k} \times$ $S_{k}$ is then the vector of probabilities of a representative STA having commenced its current MAC slot at time $k$ and it being in each state of the $\mathrm{Wi}-\mathrm{Fi}$ access process,

The possible outcomes for the representative STA during a MAC slot are: no STA transmits (noTx); other STAs transmit, but not the representative STA (othersTx); a successful transmission (success); and a collision (collision). Table I summarises the probability of occurance, duration and statedistribution-update operation for these cases when there is no interference from an LTE transmission.

TABLE I

MAC SLOT OUTCOMES, WITHOUT LTE INTEFERENCE

\begin{tabular}{r|c|c|c|c}
\hline & noTx & othersTx & success & collision \\
\hline probability & $(1-\tau)^{N}$ & $(1-\tau) p$ & $\tau(1-p)$ & $\tau p$ \\
duration & $\sigma$ & $T_{W i F i}$ & $T_{W i F i}$ & $T_{W i F i}$ \\
operation & countdown & countdown & backoff & backoff \\
& & & stage-0 & stage- $(i+1)$ \\
\hline
\end{tabular}

Let $c_{k}$ be a $\left(2^{s}-1\right) W_{0} \times 4$ matrix of conditional outcome distributions arising from MAC slots commencing at time $k$, given the representative distribution $S_{k}$, where the columns of $c_{k}$ are, in order, the probabilities of being in each state of the Wi-Fi access process after a MAC slot of type: noTx, othersTx, success and collision, given the MAC slot commenced at time $k$ with distribution $S_{k}$. As such, the elements of $c_{k}$ sum to one. Denote the elements of $c_{k}$ as $c_{k}[b, j]$, and the columns as $c_{k}[j]$. A noTx MAC slot has duration $\sigma^{u}$, and the other MAC slot types have duration $T_{W i F i}^{u}$. Let $\Delta_{j}$ denote these durations, such that $\Delta_{1}=\sigma^{u}$ and $\Delta_{2}=\Delta_{3}=\Delta_{4}=T_{W i F i}^{u}$. When an LTE transmision does not interfere with the MAC slot, applying the 'operations' in Table I gives the element of $c_{k}$ as

$$
\begin{aligned}
& c_{k}[b, 1]= \begin{cases}S_{k}[b+1]\left(1-p_{k}\right), & b \notin b^{f}, \\
0, & b \in b^{f},\end{cases} \\
& c_{k}[b, 2]= \begin{cases}S_{k}[b+1] p_{k}, & b \notin b^{f}, \\
0, & b \in b^{f},\end{cases} \\
& c_{k}[b, 3]= \begin{cases}\tau_{k} \frac{1-p_{k}}{W_{0}}, & b \in\left\{b_{0}^{0}, . ., b_{0}^{f}\right\}, \\
0, & \text { else, }\end{cases} \\
& c_{k}[b, 4]= \begin{cases}S_{k}\left[b_{i-1}^{0}\right] \frac{p_{k}}{W_{i}}, & b \in\left\{b_{i}^{0}, . ., b_{i}^{f}\right\}, i>0, \\
S_{k}\left[b_{s}^{0}\right] \frac{p_{k}}{W_{0}}, & b \in\left\{b_{0}^{0}, . ., b_{0}^{f}\right\},\end{cases}
\end{aligned}
$$

where $p_{k}$ is the collision probability at time $k$; and $\tau_{k}$ is the transmission probability at time $k$, which are evaluated as

$$
\begin{aligned}
\tau_{k} & =\sum_{i=0}^{s} S_{k}\left[b_{i}^{0}\right], \\
p_{k} & =1-\left(1-\tau_{k}\right)^{N-1} .
\end{aligned}
$$

Subsequent state distributions are then updated as

$$
\begin{aligned}
\bar{S}_{k} & =\sum_{j=1}^{4} c_{k-\Delta_{j}}[j] m_{k-\Delta_{j}}, \\
m_{k} & =\sum_{b} \bar{S}_{k}[b], \\
S_{k} & =\overline{S_{k}} / m_{k} .
\end{aligned}
$$

2) Extracting finished trajectories: Once a path reaches a successful CCA, the eNB transmits and the path returns to the starting point of 'just after an LTE transmission'. These paths have finished their trajectories for the current LTE access process and are extracted from the propagation process. The 
other paths result in an unsuccessful CAA, and they continue. The final state distributions of the extracted paths contribute to the initial state distribution of the next LTE access process.

To identify the paths that have reached a successful CCA define sets of times: $K_{u}, K_{n}$ and $K_{e}$ as

$$
\begin{aligned}
K_{u} & =\left\{T_{\text {Idle }}^{u}-\delta^{u}+1, \ldots, T_{\text {Idle }}^{u}+\delta^{u}\right\} \\
K_{n} & =\left\{T_{\text {Idle }}^{u}+\delta^{u}+1, . ., T_{\text {Idle }}^{u}+D I F S^{u}-T_{C C A}^{u}\right\} \\
K_{e} & =K_{u} \cup K_{n}
\end{aligned}
$$

When a MAC slot is due to commence during any count $k=$ $k^{\prime}+(r-1) T_{F F P}^{u}$, such that $k \bmod T_{F F P}^{u} \in K_{e}$, it can be inferred that the CCA ending on count $T_{\text {Idle }}^{u}+(r-1) T_{F F P}^{u}$ would have found, or will find, the channel 'clear'. Depending on the timing, the Wi-Fi state is updated at count $k$, or not.

During counts $k$, for which $k \bmod T_{F F P}^{u} \in K_{u}$, a new MAC slot will commence and the STAs will update their access processes to state $S_{k}$ before sensing the eNB transmission. Since the eNB will transmit during the MAC slot, the representative STA will either have a MAC slot outcome at count $k$ of type othersTx or collision, with conditional outcome distributions, given state $S_{k}$, denoted $c_{k}^{K_{u}}$, given by

$$
\begin{aligned}
c_{k}^{K_{u}}[b, j] & =0, \quad j \in\{1,3\}, \\
c_{k}^{K_{u}}[b, 2] & =c_{k}[b, 1]+c_{k}[b, 2], \\
c_{k}^{K_{u}}[b, 4] & = \begin{cases}S_{k}\left[b_{i-1}^{0}\right] \frac{1}{W_{i}}, & b \in\left\{b_{i}^{0}, . ., b_{i}^{f}\right\}, i>0, \\
S_{k}\left[b_{s}^{0}\right] \frac{1}{W_{0}}, & b \in\left\{b_{0}^{0}, . ., b_{0}^{f}\right\} .\end{cases}
\end{aligned}
$$

During counts $k$, for which $k \bmod T_{F F P}^{u} \in K_{n}$, the LTE block transmission will already be audible to the STAs, so the STAs 'freeze' their backoff access processes and no new MAC slot occurs. The weighted conditional outcome distributions of the paths that leave the propagation process are those from the previous MAC slots commencing at times $k-\Delta_{j}$ that were due to be updated at time $k$.

The marginal state distribution of the paths extracted at count $k$ during the $r^{t h} \mathrm{CCA}$ is denoted $e_{k}^{r}$ and is given by

$$
e_{k}^{r}= \begin{cases}\sum_{j \in\{2,4\}} c_{k}^{K_{u}}[j] m_{k}, & k-(r-1) T_{F F P}^{u} \in K_{u}, \\ \sum_{j=1}^{4} c_{k-\Delta_{j}}[j] m_{k-\Delta_{j}}, & k-(r-1) T_{F F P}^{u} \in K_{n} .\end{cases}
$$

All the paths from counts $k$, for which $k \bmod T_{F F P}^{u} \in K_{e}$, leave the propagation process, so after extracting these paths, their probability masses, $m_{k}$ are set to 0 and the propagation process continues.

The process is propagated for $R$ FFPs, then $P_{C C}(r)$ is evaluated directly from $e_{k}^{r}$ as

$$
P_{C C}(r)=\sum_{k \in K_{e}}\left\|e_{k+(r-1) T_{F F P}^{u}}^{r}\right\|_{1}, \quad r \in\{1, . . R\},
$$

where $\left\|e_{k}^{r}\right\|_{1}$ is the 1-norm of $e_{k}^{r} \cdot P_{C C}$ is then obtained from (9)-(11).

3) Calculating the initial distribution: The initial distribution, $S_{1}$, is the sum of the marginal state distributions from the final MAC slots of the many different paths that may have started after different LTE transmissions but all return on the same LTE transmission.

For an initial estimate of $S_{1}$, the steady-state distribution of the $N$ STA network can be used. This is obtained by simultaneously solving (2) and (3), then setting $S_{1}$ to $\tilde{S}_{1} /\left\|\tilde{S}_{1}\right\|_{1}$, where

$$
\tilde{S}_{1}[b]=p^{i} \frac{b_{i}^{f}-b+1}{W_{i}}, b \in\left\{b_{i}^{0}, . ., b_{i}^{f}\right\}, 0 \leq i \leq s .
$$

The estimate of $S_{1}$ is updated from all the extracted path contributions, $e_{k}^{r}$, obtained over the $R$ FFPs as

$$
\begin{aligned}
S_{1}= & \sum_{r=1}^{R} \sum_{k \in K_{e}} e_{k+(r-1) T_{F F P}^{u}}^{r}+ \\
& \frac{\left(1-\sum_{r=1}^{R} P_{C C}(r)\right)}{P_{C C}(R)} \sum_{k \in K_{e}} e_{k+(r-1) T_{F F P}^{u}}^{R},
\end{aligned}
$$

and the whole propagation process is repeated until $P_{C C}$ converges. The $P_{C C}$ estimate usually converges to within $1 \%$ of its final value after the second iteration of the whole propagation process and convergence to within $0.05 \%$ is usually achieved within 4 iterations. (9)-(31) and (1) are combined to form an algorithm for calculating $P_{C C}$ and $\rho_{L T E}$ in Algorithm 1.

4) LTE/Wi-Fi collisions: As mentioned, if a Wi-Fi STA is due to commence a MAC slot during any count $k$ $\bmod T_{F F P}^{u} \in K_{u}$ it will update its backoff process before sensing the eNB transmision. If this update causes the STA to transmit, there will be an LTE/Wi-Fi collision. The probability of at least one STA transmitting at count $k$ is $m_{k}\left(1-\left(1-\tau_{k}\right)^{N}\right)$, so the probability of an LTE transmission being an LTE/Wi-Fi collision, $p_{L}$, is estimated as

$$
\begin{gathered}
p_{L}=\sum_{r=1}^{R} \sum_{\left\{k \mid k-(r-1) T_{F F P}^{u} \in K_{u}\right\}} m_{k}\left(1-\left(1-\tau_{k}\right)^{N}\right)+ \\
\left(1-\sum_{r=1}^{R} P_{C C}\right) p_{L}^{s s}
\end{gathered}
$$

where $P_{L}^{s s}$ is the Steady-state model value for $p_{L}$, obatained from (7) in Section III-A. The double sum models the collisions from the first $R$ CCAs with the dynamic model and the second term models the collisions from any further CCAs with the steady-state model.

\section{Throughput Calculations}

To make throughput calculations, we assume the eNB used the Type-1 LTE frame structure for FB-LBT access. The Type1 LTE frame has an overall length of $10 \mathrm{~ms}$, which is divided into 10 subframes, each one millisecond long. Each subframe is further divided into 14 slots, and each slot supports one orthogonal frequency-division multiplexing (OFDM) symbol. Control signalling for each subframe occurs at the start of each subframe. As such, the LTE subframes that collide with Wi-Fi transmissions are not decoded and the remaining subframes are decoded.

The control format indicator (CFI) indicates the number of OFDM symbols used for control each subframe. The initial 


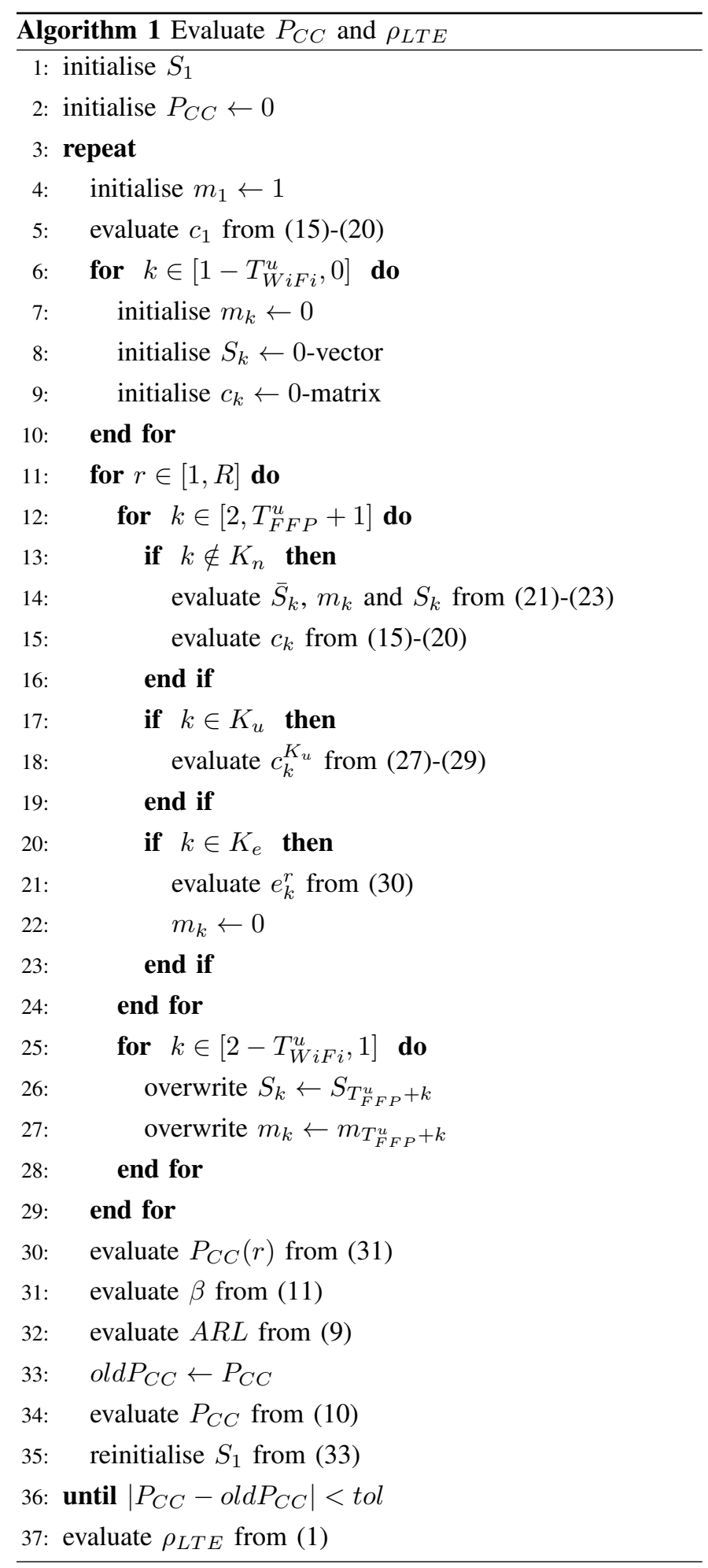

CFI OFDM symbols of each subframe are used for control [21], [22], and the remainder are used to transmit data. The CFI can take value 1,2 , or 3 . We use $\mathrm{CFI}=2$, on average. Let $\epsilon_{L}$ denote the proportion of LTE channel time spent transmitting data, so that $\epsilon_{L}=1-\mathrm{CFI} / 14$, and let $r_{L}$ denote the LTE data transmission rate. Then the overall LTE throughput, denoted $\operatorname{Thr}(L T E)$, can be calculated as

$$
\operatorname{Thr}(L T E)=r_{L} \times \epsilon_{L} \times \rho_{L T E}\left(1-\frac{\left\lceil 10^{3} \times T_{W i F i}\right\rceil}{10^{3} \times T_{L T E}} p_{L}\right)
$$

where $\lceil$.$\rceil is the ceiling function, rounding up to the nearest$ integer.

During the Idle Period, when the Wi-Fi STAs control the channel, the Wi-Fi throughput equals the payload/Wi-Fi packet times the number of successfully transmitted Wi-Fi packets/second. The proportion of time Wi-Fi controls the channel is $1-\rho_{L T E}$, so, the overall Wi-Fi throughput, denoted $\operatorname{Thr}(W i F i)$, can be estimated from (1)-(5) as

$$
\operatorname{Thr}(W i F i)=L_{W} \frac{N \tau(1-p)}{E_{s}}\left(1-\rho_{L T E}\right),
$$

where $L_{W}$ is the payload per Wi-Fi packet.

\section{FB-LBT Coexistence Performance AND Limits}

The coexistence network was simulated via an event-driven simulation, where a separate access process was maintained for each competing device (WiFi Station or LTE eNB). The event-driven simulation software package was coded in $\mathrm{R}$ [25], which is a powerful language (and in many ways similar to Matlab). The simulations reproduce all the contention and collision details of the CSMA/CA and LBT processes, which are essential parts of the coexistence operations.

The Steady-state and Dynamic models developed in Section III were validated against the event-driven simulations for a Wi-Fi/LTE coexistence scenario. We consider an indoor office environment in which LTE and Wi-Fi traffic are competing for the same $20 \mathrm{MHz}$ channel, with centre frequency $5.2 \mathrm{GHz}$. The office is single-floored, open-plan, with area 35 $\mathrm{m} \times 35 \mathrm{~m}$. A small-cell eNB and a Wi-Fi access point (AP) are located in the centre of the office floor.

The small cell operates under the FB-LBT protocol of Section II-A and supports LTE traffic for a number of UE using a Type-1 LTE frame structure and a data rate of $100 \mathrm{Mbps}$. The number of UE is unspecified, however the CFI is assumed to equal two, and the LTE traffic is assumed saturated, so that the eNB transmits a $10 \mathrm{~ms}$ frame each successful channel access.

The AP supports Wi-Fi traffic resulting in a total of $N$ saturated backoff entities, which are either the AP or a Wi-Fi station (STA). $N=1$ represents an AP transmitting in DL; $N=2$ represents the AP and one STA; and $N=10$ represents the AP and 9 backoff entities. The Wi-Fi traffic operates under the IEEE 802.11n protocol, using a single spatial stream in the $20 \mathrm{MHz}$ channel, with modulation and coding scheme (MCS) Index 7 (i.e. 64-QAM and 5/6 coding rate), unless specified otherwise.

The Wi-Fi devices decode other Wi-Fi transmissions above $-82 \mathrm{dBm}$, using carrier sensing, and detect LTE transmissions 
above $-62 \mathrm{dBm}$, using energy detection. The LTE devices decode other LTE transmissions above their receiver sensitivities, e.g., $-90 \mathrm{dBm}$, and detect Wi-Fi transmissions above $-75 \mathrm{dBm}$, during the CCA [10]. So, all devices can detect each other when the receiver power exceeds $-62 \mathrm{dBm}$. The transmission power of all devices is taken to be $24 \mathrm{dBm}$.

Line-of-sight (LOS) and non-LOS attenuation models for an indoor hotspot $(\mathrm{InH})$ were obtained from Table B.1.2.1-1 and Table B.1.2.1-2 of [26]. The LOS and non-LOS models have $3 \mathrm{~dB}$ and $4 \mathrm{~dB}$ shadow fading respectively and Table B.1.2.12 of [26] prescribes the probability of using each model at a given distance. The largest distance between two Wi-Fi devices in the simulation is $35 \sqrt{2} \mathrm{~m}=49.5 \mathrm{~m}$, and the largest distance between the eNB and a Wi-Fi device $35 \sqrt{2} \mathrm{~m}=24.7 \mathrm{~m}$.

The 'between Wi-Fi and eNB' probability of the receiver power dropping below $-62 \mathrm{dBm}$ was calculated as 0.004 by combining the models from [26] with an equation for the area of intersection between an annulus and a square. The probability of the receiver power dropping below $-82 \mathrm{dBm}$ for transmissions between two Wi-Fi stations was calculated numerically as $4 \times 10^{-5}$; between the Wi-Fi AP and a WiFi station, the probability of being inaudible is considerably lower. As such, the simulation is performed with perfect channel sensing.

The default system parameters are summarized in Table II, giving $T_{W i F i}=254 \mu \mathrm{s}$ and $T_{L T E}=10 \mathrm{~ms}$. $T_{W i F i}$ is calculated from the parameters in Table II as

$$
\begin{aligned}
T_{W i F i}= & \text { preamble }+(\text { header }+ \text { payload }) / \text { rate }+ \\
& \text { SIFS }+ \text { ACK }+ \text { DIFS }
\end{aligned}
$$

So, for $802.11 \mathrm{n}(20)$,

$$
\begin{aligned}
T_{W i F i}^{.11 \mathrm{n}(20 \mathrm{MHz})}= & 20+(64 \times 8+1460 \times 8) / 72.2+ \\
& 16+15.5+34 \mu \mathrm{s} \\
= & 85.5+12192 / 72.2 \mu \mathrm{s} \\
= & 254 \mu \mathrm{s} .
\end{aligned}
$$

TABLE II

SIMULATION SETTINGS

\begin{tabular}{rr|rr}
\hline \multicolumn{2}{r|}{ Channel Occupancy Time } & & $10 \mathrm{~ms}$ \\
Idle Period & & $0.5 \sim 7 \mathrm{~ms}$ \\
& $T_{C C A}$ & & $20 \mu \mathrm{s}$ \\
\hline Slot time $\sigma$ & $9 \mu \mathrm{s}$ & Preamble & $20 \mu \mathrm{s}$ \\
SIFS & $16 \mu \mathrm{s}$ & Wi-Fi data rate & $72.2 \mathrm{Mbps}$ \\
DIFS & $34 \mu \mathrm{s}$ & Wi-Fi headers & 64 byte \\
$W_{0}$ & 16 & Wi-Fi payload & 1460 byte \\
$W_{m}$ & 512 & ACK & $15.5 \mu \mathrm{s}$ \\
\hline
\end{tabular}

\section{A. Model Validation}

The Steady-state and Dynamic model estimates of $P_{C C}$ are plotted against $T_{\text {Idle }}$ for $N \in\{1,2,10\}$ in Fig. 2 and are compared to simulation values. $T_{W i F i}$ was set to $254 \mu \mathrm{s}$, representing the $\mathrm{Wi}-\mathrm{Fi}$ transmission time of a 1460 byte packet for $802.11 \mathrm{n}(20 \mathrm{MHz})$, and the number of FFPs in the simulation, denoted $N_{F F P}$, was set to 25,000 . The dashed horizontal lines are the Steady-state model estimates of $P_{C C}$, which are indepedent of $T_{I d l e}$; the oscillating solid lines are the Dynamic model estimates of $P_{C C}$; and the solid circles with capped vertical bars are the simulation values and $95 \%$ confidence intervals. The $95 \%$ Confidence Intervals, denoted $C I_{95 \%}$, were calculated as

$$
C I_{95 \%}=b \pm z_{0.975} \sqrt{\frac{b(1-b)}{N_{F F P}}},
$$

where $b$ represents a particular simulation estimate of $P_{C C}$; and $z_{0.975}$ is the $97.5 \%$ quantile from the standard normal distribution. $b$ was calculated as $b=N_{\text {clear }} / N_{F F P}$, where $N_{\text {clear }}$ is the number of CCAs that find the channel 'clear' during the simulation.

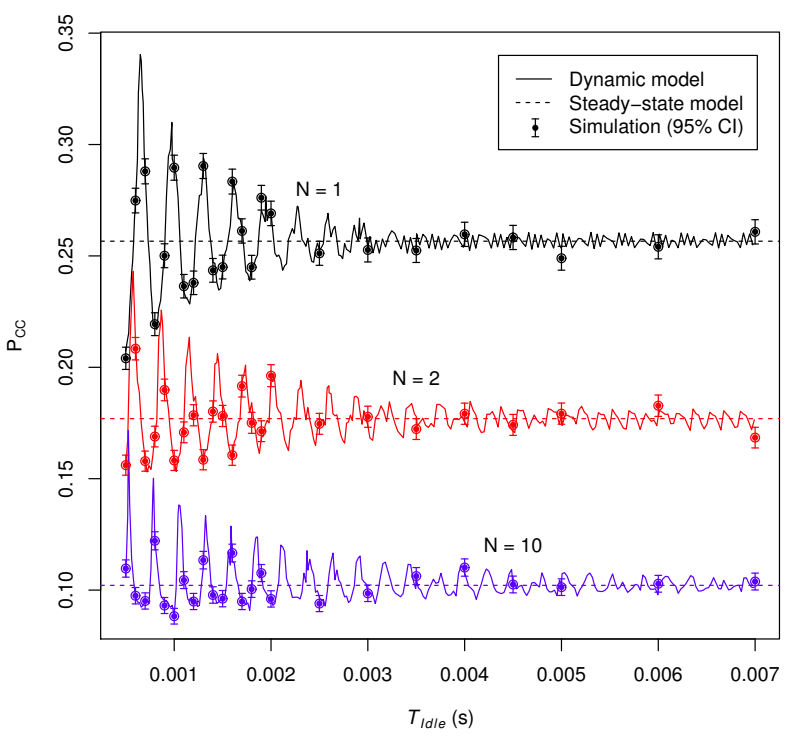

Fig. 2. Model validation: $P_{C C}$ vs $T_{I d l e}$ for $N \in\{1,2,10\} ; T_{W i F i}=$ $254 \mu s ; N_{F F P}=25,000$.

The Dynamic-model estimates of $P_{C C}$ agree with the simulation values to a high level of accuarcy at almost every simulation point evaluated. The simulation results, which look somewhat ad-hoc when taken in isolation, are revealed as belonging to damped oscillating patterns by the Dynamic model. The Steady-state model estimates are close to the means of the oscillating patterns and, as the Idle Period is increased, the Dynamic-model estimates converge to the Steady-state-model estimates.

For $N=10$, the first Dynamic-model $P_{C C}$-estimate peak is $65 \%$ higher than the Steady-state model estimate; and for $N=1$, the first Dynamic-model $P_{C C}$-estimate trough is $19 \%$ lower than the Steady-state model estimate. When $T_{\text {Idle }} \gtrsim 2$ $\mathrm{ms}, 3 \mathrm{~ms}$ and $4 \mathrm{~ms}$, for $N=1,2$, and 10 respectively, the deviation of the Dynamic-model oscillation peaks from the Steady-state model values are within $5 \%$. This corresponds to $T_{\text {Idle }} \gtrsim 8 T_{W i F i}, 12 T_{W i F i}$ and $16 T_{W i F i}$.

The periodicties of the oscillations, for $N=1,2$ and 10 , are approximately $322 \mu s, 288 \mu s$ and $261 \mu s$, which are 
also approximately half the locations of the first peaks. The periodicity is explored in Section IV-E.

\section{B. LTE Spectrum Share}

The proportion of channel time used by the eNB, $\rho_{L T E}$, was evaluated for a range of $T_{\text {Idle }}$ and for $N \in\{1,2,10\}$, using the validated models, with $T_{W i F i}=254 \mu \mathrm{s}$, The results are shown in Fig. 3. As for Fig. 2, the dashed lines are estimates from the Steady-state model and the oscillating solid lines are estimates from the Dynamic model.

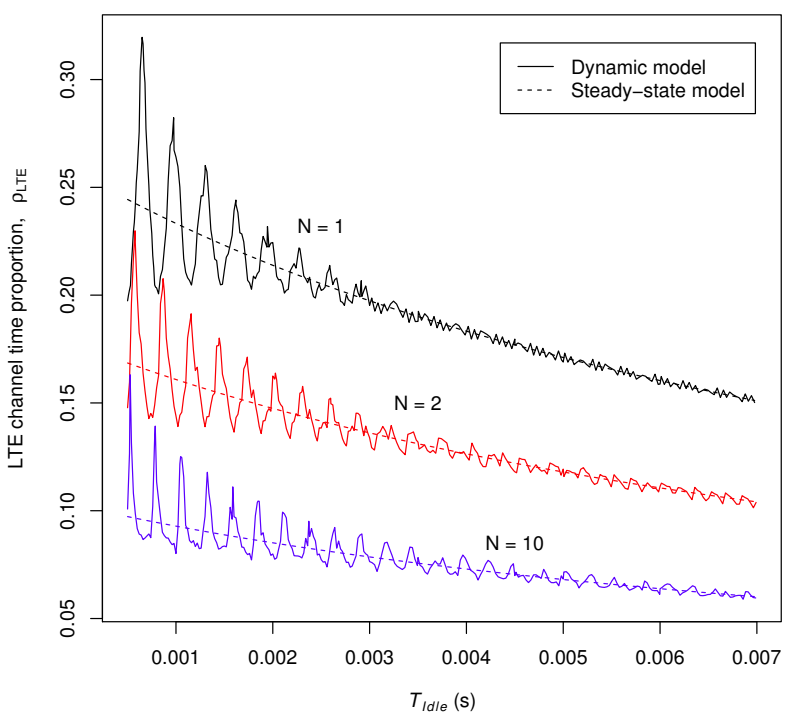

Fig. 3. LTE Channel time proportion $\left(\rho_{L T E}\right)$ vs $T_{\text {Idle }}$ for $N \in\{1,2,10\}$; $T_{W i F i}=254 \mu \mathrm{s}$.

Since $\rho_{L T E}$ is proportional to $P_{C C}$ and inversely proportional to $T_{F F P}$, the same oscillating pattern in Fig. 2 is apparent, and the Dynamic-model estimates has the same percentage deviation from the Steady-state-model estimates.

With $T_{W i F i}=254 \mu \mathrm{s}$, the maximum proportion of time the eNB can access the channel is limited to $32 \%$, which is achieved with $T_{\text {Idle }}=650 \mu \mathrm{s}$, when $N=1$. As presented in Section IV-F, the location and value of the maximum $\rho_{L T E}$ is dependent on $T_{W i F i}$, which depends on the Wi-Fi payload and Wi-Fi technology.

It can be concluded from Fig. 3 that this FB-LBT scheme is 'friendly' to Wi-Fi in the sense that it does not take an unfair portion of the channel time. A single Wi-Fi STA, or backoff entity, still obtains $68 \%$, and possibly $75 \%-80 \%$, if the LTE settings are not precise. With $10 \mathrm{Wi}-\mathrm{Fi}$ STAs, or backoff entities, Wi-Fi obtains more than $84 \%$, and possibly above $90 \%$. Noting that the eNB generally serves multiple UE, Wi$\mathrm{Fi}$ is still obtaining an unfair portion of the channel share.

\section{Throughput}

The average LTE and Wi-Fi throughputs, $\operatorname{Thr}(L T E)$ and $\operatorname{Thr}(W i F i)$, were calculated as per Section III-C, with $\rho_{L T E}$ evaluated from the dynamic model, for $N \in\{1,2,10\}$, over a range of $T_{I d l e}$. The Wi-Fi packets had $L_{W}=$ 1460 byte payloads that were transmitted at 72.2 Mbps using $802.11 \mathrm{n}(20 \mathrm{MHz})$, so that $T_{W i F i}=254 \mu \mathrm{s}$. The LTE transmissions were $10 \mathrm{~ms}$, with data rate $r_{L}=100 \mathrm{Mbps}$ and $\epsilon_{L}=0.95$, so that the technology is comparable to the $802.11 \mathrm{n}(20 \mathrm{MHz})$ technology.

The resulting $\operatorname{Thr}(L T E)$ and $\operatorname{Thr}(W i F i)$ are plotted against $T_{\text {Idle }}$ in Fig. 4. The solid lines are $\operatorname{Thr}(L T E)$ and the dashed lines are $\operatorname{Thr}(W i F i)$. $\operatorname{Thr}(L T E)$ is proportional to $\rho_{L T E}$, so the solid lines have the same oscillating pattern as in Fig. 3. $\operatorname{Thr}(W i F i)$ is is proportional to $1-\rho_{L T E}$, so the dashed lines have a complimentary oscillating pattern to $\operatorname{Thr}(L T E)$.

The dashed $\operatorname{Thr}(W i F i)$ lines, from the top of the figure, are for $N=2,10$ and 1 . This non-sequential ordering occurs because $N=2$ allows more Wi-Fi transmissions than $N=1$, while not causing many collisions; whereas, for more than two Wi-Fi STAs, although increasing $N$ produces more transmissions, it also leads to more collisions, with the net effect of reducing the average Wi-Fi throughput. The order of all the lines, top to bottom, is the same as in the legend.

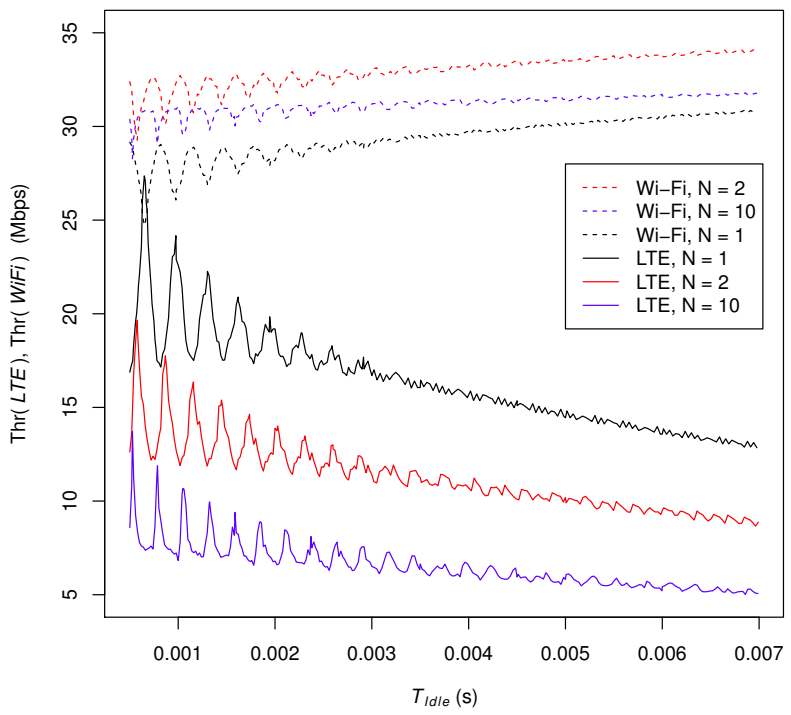

Fig. 4. Throuhgput, $\operatorname{Thr}(L T E)$ and $\operatorname{Thr}(W i F i)$, vs $T_{I d l e}$, for $N \in$ $\{1,2,10\} ; T_{L T E}=10 \mathrm{~ms}$, transmitting at $100 \mathrm{Mbps} ; T_{W i F i}=254 \mu \mathrm{s}$, under $802.11 \mathrm{n}(20 \mathrm{MHz})$ with 1460 byte payload per packet.

\section{Comparison to other approaches}

We next compare the performance of FB-LBT with a dutycycle approach and a 'traditional' LBT approach. In the dutycycle approach, the eNB uses fixed 'on' and 'off' periods. The structure is similar to the FB-LBT structure shown in Fig. 1 but, rather than performing a CCA to determine whether to transmit each FFP, the eNB always begins a transmission at the start of each FFP. In the traditional LBT approach, the eNB uses the traditional Wi-Fi CSMA/CA process to determine channel access, and once access is obtained, the eNB transmits a full $10 \mathrm{~ms}$ frame. 
The alternative approaches were assessed via simulation. The Wi-Fi and LTE throughput are presented in Fig. 5 for the duty-cycle and traditional LBT approaches; and the LTE collision probability, $p_{L}$, is presented in Fig. 6 for the dutycycle, traditional LBT and FB-LBT approaches.

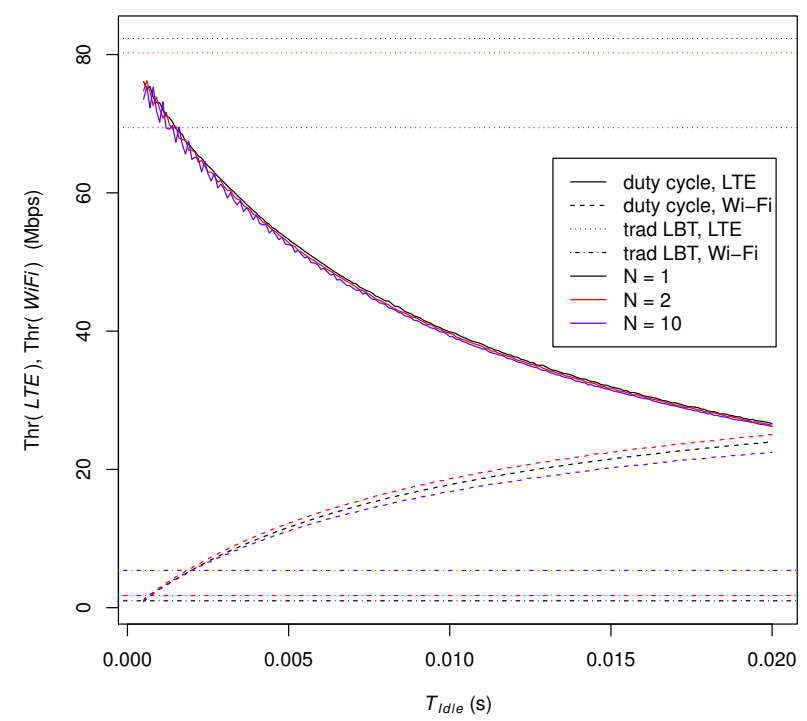

Fig. 5. Throuhgput for alternative approaches, $\operatorname{Thr}(L T E)$ and $\operatorname{Th}(\mathrm{WiFi})$, vs. $T_{\text {Idle }}$, for duty cycle and 'traditional' LBT approaches. $N \in 1,2,10$; $T_{L T E}=10 \mathrm{~ms}$, transmitting at $100 \mathrm{Mbps} ; T_{W i F i}=254 \mu \mathrm{s}$, under $802.11 \mathrm{n}(20 \mathrm{MHz})$ with 1460 byte payload per packet.

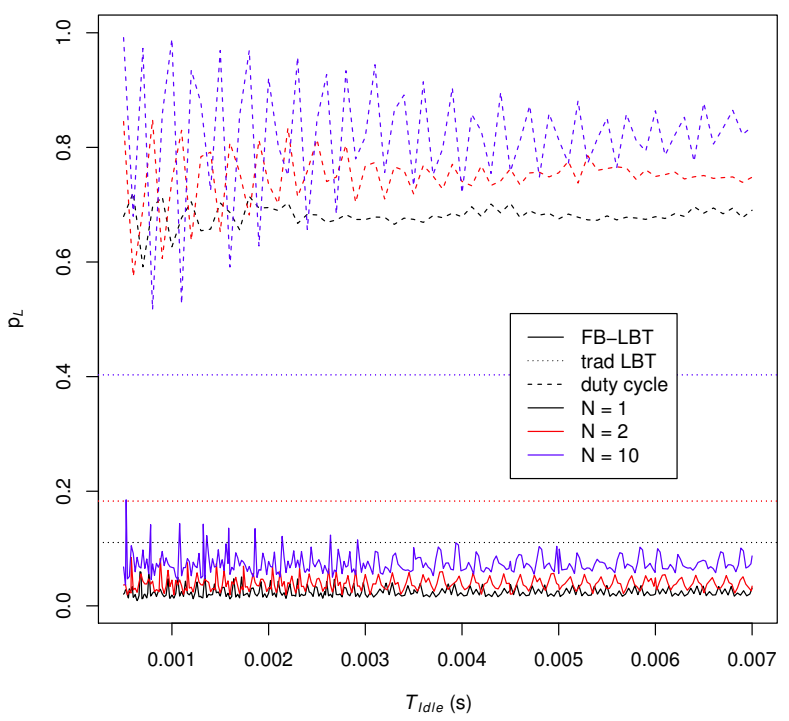

Fig. 6. LTE collision probability comparison, $p_{L}$ vs. $T_{I d l e}$, for FB-LBT, duty cycle and 'traditional' LBT approaches. $N \in 1,2,10 ; T_{L T E}=10 \mathrm{~ms}$, transmitting at $100 \mathrm{Mbps} ; T_{W i F i}=254 \mu \mathrm{s}$, under $802.11 \mathrm{n}(20 \mathrm{MHz})$ with 1460 byte payload per packet.

The traditional LBT approach does not have a set time for $T_{\text {Idle }}$, so the results are presented as horizontal lines in both
Fig. 5 and Fig. 6. Dotted lines as used for LTE and dotdash lines for Wi-Fi, with (Black, red, blue) corresponding to $(1,2,10)$ competing Wi-Fi stations. For the traditional LBT approach, all nodes use the same CSMA/CA process, so each node (LTE or Wi-Fi) has approximately the same collision probability and hence has throughput approximately proportional its payload per transmission. The LTE transmissions are substantially longer, so $\operatorname{Thr}(L T E)$ is substantially higher than $\operatorname{Thr}(W i F i)$. LTE also has the advantage of only losing collided subframes, rather than the whole frame, making $T h r(L T E)$ relatively higher again. From Fig. $6, p_{L} \approx 0.4$ for $N=10$, so this second advantage is a substantial factor. With $T_{L T E}=10 \mathrm{~ms}$ and $T_{W i F i}=254 \mu \mathrm{s}$, the traditional LBT approach is very unfair to Wi-Fi, especially when $N=1$.

For the duty-cycle approach, $\operatorname{Thr}(L T E)$ and $T h r(W i F i)$ respectively fall and rise with $T_{\text {Idle }}$ (solid lines for LTE and dashed lines for $\mathrm{Wi}-\mathrm{Fi}$ ). There is a slight oscillatory effect, but the effect is much less pronounced than for FB-LBT throughput, as is apparent in Fig. 4. A much stronger oscillatory effect can be seen for $p_{L}$ in Fig. 6 (dashed lines), for the duty-cycle approach. The effect of these strong oscillations in $p_{L}$ on the duty-cycle $\operatorname{Thr}(L T E)$ is secondary, when $T_{W i F i}$ $=254 \mu \mathrm{s}$, because $p_{L}$ only affects the success of the first LTE subframe. The corresponding effect on FB-LBT is to cause unsuccessful CCAs and hence cause completely missed LTE transmissions. That is, $p_{L}$ for the duty-cycle approach is approximately $\left(1-P_{C C}\right)$ as calculated for FB-LBT, which is the complement of Fig. 2.

For the same $T_{\text {Idle }}, \operatorname{Thr}(L T E)$ is substantially higher for the duty-cycle approach (Fig. 5) than for FB-LBT (Fig. 4). This provides the duty-cycle approach with a wider dynamic range of throughput and $\rho_{L T E}$, noting that $\rho_{L T E}=$ $T_{L T E} /\left(T_{L T E}+T_{I d l e}\right)$ for the duty-cycle approach. For the $T_{\text {Idle }}$ domain in Fig. 5, the duty-cycle $\rho_{L T E}$ ranges from 95\% down to $33 \%$. The negative aspect of the duty-cycle approach, however, is that the LTE collision probability is very high, such that the first LTE subframe is lost approximately $70-80$ percent of the time.

\section{E. First Oscillation Peak Location}

The oscillations in $P_{C C}$ seen in Fig. 2 are periodic, with peaks approximately located at integer multiples of the period. Let $T\left(N, T_{W i F i}\right)$ be the oscillation period of $P_{C C}$ as a function of $T_{I d l e}$, for $N$ saturated Wi-Fi STAs transmitting with packet duration $T_{W i F i}$. The periodicty $T\left(N, T_{W i F i}\right)$ can be approximated by $\hat{T}\left(N, T_{W i F i}\right)$, where

$$
\hat{T}\left(N, T_{W i F i}\right)=T_{W i F i}+\frac{W_{0}-1}{2 N} \sigma .
$$

Let $f\left(N, T_{W i F i}\right)$ be the lowest permissible $T_{\text {Idle }}$ that produces a $\rho_{L T E}$ maximum, noting that $T_{I d l e}$ is prescribed to be no less than $0.05 T_{L T E}$. Then $f\left(N, T_{W i F i}\right)$, is approximately given by $\hat{f}\left(N, T_{W i F i}\right)$, where

$$
\begin{array}{r}
\hat{f}\left(N, T_{W i F i}\right)=\min \left\{k \hat{T}\left(N, T_{W i F i}\right) \mid k \in \mathbb{N},\right. \\
\left.T_{I d l e} \geq 0.05 T_{L T E}\right\},
\end{array}
$$

and $\mathbb{N}$ is the set of natural numbers. The accuracy of this approximation to $f\left(N, T_{W i F i}\right)$ is now considered. 
Fig. 7 plots $f\left(N, T_{W i F i}\right)$ vs $T_{W i F i}$, for $N=10$. The markers are estimates from the Dynamic model, obtained by maximising the Dynamic model's $P_{C C}$. The solid lines are $\hat{f}\left(N, T_{W i F i}\right)$.

The fluctuations in the Dynamic-model peaks are caused by small deviations in $P_{C C}$ that are in addition to the main oscillatory pattern and have locally repeating patterns with periodicty $\sigma$. The lower limit of $500 \mu \mathrm{s}$ is due to $T_{\text {Idle }}$ being prescribed as greater than $5 \%$ of the Channel Occupancy Time, which has been set at $10 \mathrm{~ms}$. The discontinuities are a result of the multiplier $k$ that minimises $\hat{f}\left(N, T_{W i F i}\right)$ in (41) changing from 2 to 6 , going from right to left. $\hat{f}\left(N, T_{W i F i}\right)$ closely approximates $f\left(N, T_{W i F i}\right)$, with little phase error. If $\hat{f}\left(N, T_{W i F i}\right)$ were used to locate peaks in $\rho_{L T E}$ for much higher $T_{\text {Idle }}$, for example the $20^{\text {th }}$ peak, the phase error would accumulate and make the peak location estimates unreliable. However, this is also the region where the magnitude of the oscillations are relatively small, so the deviations of $\rho_{L T E}$ from the steady-state model will also be small.

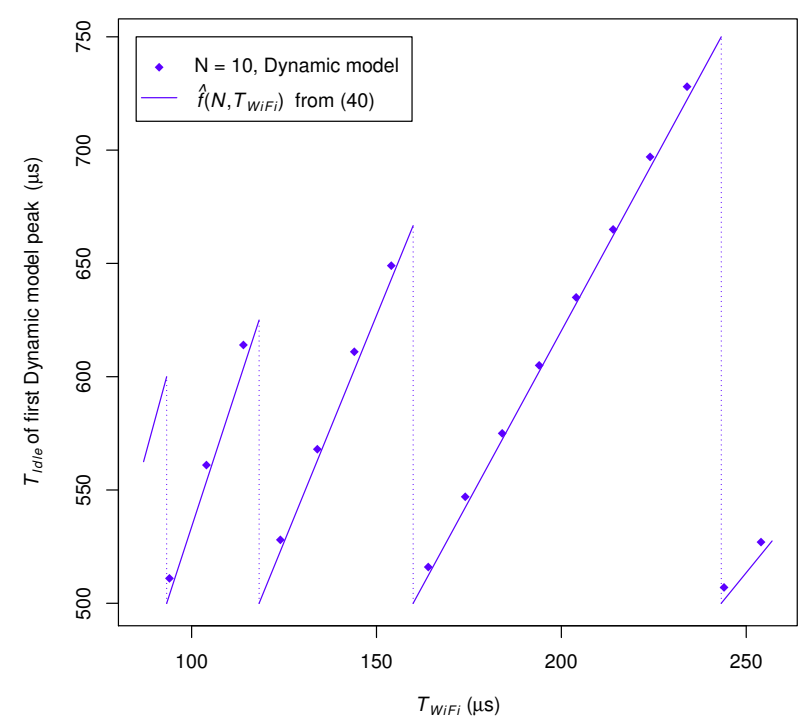

Fig. 7. Lowest permissible $T_{\text {Idle }}$ that produces an LTE Channel time proportion peak vs $T_{W i F i}$ for $N=10$.

\section{F. Limits of LTE Spectrum Share}

We investigate the maximum spectrum share that LTE can aquire when coexisting with various Wi-Fi technologies that can operate in the $5 \mathrm{GHz}$ unlicenced spectrum. The analysis in Section IV-B shows that the maximum spectrum share $\rho_{L T E}$ occurs with $N=1$ at the $T_{\text {Idle }}$ that produces the first oscillation peak ${ }^{2}$, where $T_{\text {Idle }} \geq 500 \mu \mathrm{s}$. We now consider the scenario where a single Wi-Fi device continuously transmits packets with 1460 byte payloads. Such a scenario typically happens, for example, when a single user downloads a long streaming video in an otherwise quiet Wi-Fi network.

\footnotetext{
${ }^{2}$ The maximum $\rho_{L T E}$ may occur near $500 \mu s$ when the first peak is located at almost a whole oscillation period beyond the minimum $T_{\text {Idle }}$ of $500 \mu \mathrm{s}$.
}

TABLE III

TRANSMISSION TIMES AND MAXIMUM $\rho_{L T E}$

\begin{tabular}{l|c|c|c|c|c}
\hline $\begin{array}{l}\text { Network } \\
\text { Technology }\end{array}$ & $\begin{array}{c}\text { preamble } \\
(\mu s)\end{array}$ & $\begin{array}{c}\text { data rate } \\
(\mathrm{Mbps})\end{array}$ & $\begin{array}{c}\text { ACK } \\
(\mu s)\end{array}$ & $\begin{array}{c}T_{W i F i} \\
(\mu s)\end{array}$ & $\begin{array}{c}\max \\
\rho_{L T E}\end{array}$ \\
\hline $802.11 \mathrm{n}(20 \mathrm{MHz})$ & 20 & 72.2 & 15.5 & 254 & 0.320 \\
$802.11 \mathrm{n}(40 \mathrm{MHz})$ & 36 & 150 & 7.5 & 175 & 0.384 \\
$802.11 \mathrm{ac}(80 \mathrm{MHz})$ & 40 & 433.3 & 3.5 & 122 & 0.425 \\
$802.11 \mathrm{ac}(160 \mathrm{MHz})$ & 40 & 866 & 1.7 & 106 & 0.463 \\
\hline
\end{tabular}

The transmission rates for the different Wi-Fi technologies considered are given in Table III, along with the resulting $T_{W i F i}$, calculated from (37) for 1460-byte Wi-Fi payloads per packet, and the maximum $\rho_{L T E}$ obtained for $T_{\text {Idle }} \geq 500 \mu \mathrm{s}$.

As $T_{W i F i}$ shortens, the maximum $\rho_{L T E}$ increases. This is a consequence of the CSMA/CA backoff process having the same slot time, SIFS and DIFS, for all the Wi-Fi technologies considered. As such, as the data rate increases, the time spent transmitting each Wi-Fi packet decreases, while the silent time between transmissions remains unchanged, so the proportion of time the channel is silent increases, which increases the chances of successful CCAs.

\section{G. Controlling Access Time}

Our models presented in Section III can be exploited to control the spectrum share for LTE access. In particular, the eNB can select $T_{\text {Idle }}$ to achieve a desired LTE spectrum share, based on Wi-Fi network traffic conditions in terms of the number of active STAs, $N$, and their transmission duration, $T_{W i F i}$. In practice, these conditions can be estimated by listening in on the Wi-Fi channel with a Wi-Fi sniffer.

The traffic conditions can be categorised as having, 1) a clearly dominant $\left.T_{W i F i} ; 2\right)$ a few dominant transmission sizes; or 3) no clear pattern. The approaches to controlling the spectrum share are then:

1) When there is a clearly dominant $T_{W i F i}$, the eNB can use the results for the Dynamic model to select a $T_{\text {Idle }}$ that produces the target $\rho_{L T E}$. One option is for the eNB to run Algorithm 1, using the $N$ and $T_{W i F i}$ estimated by the Wi-Fi sniffer, with $T_{L T E}=10 \mathrm{~ms}$, and searching for a $T_{I d l e}$ that produces the target $\rho_{L T E}$, noting that $T_{F F P}=T_{L T E}+T_{\text {Idle }}$. Another option is to run Algorithm 1 off-line on a selection of $N$ and $T_{W i F i}$, over a range of $T_{\text {Idle }}$, with $T_{L T E}=10 \mathrm{~ms}$, to create a lookup table, based on Fig. 3, that the eNB

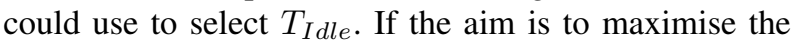
eNB's spectrum share, $T_{\text {Idle }}$ could be dynamically set to $\hat{f}\left(N, T_{W i F i}\right)$, as per (40) and (41).

2) When there are a few dominant transmission times, the resulting $\rho_{L T E}$ is expected to be an interpolation between the $\rho_{L T E}$ obtained at each dominant transmission time, for the selected $T_{\text {Idle }}$. As such, an approach that is robust to how this mixing occurs is for the eNB to choose a $T_{\text {Idle }}$ that produces similar $\rho_{L T E}$ for each of the dominant transmission times.

3) When there is no clear pattern in the transmission times, it is expected that there will be a less rigid Wi-Fi MAC- 
slot timing structure so that $T_{\text {Idle }}$ will be approximately given by the Steady-state model with $T_{W i F i}$ set to the average transmission time. This approach is based on the following conjecture: that the oscillatory patterns in $\rho_{L T E}$ occur primarily because there is still substantial structure in the timing of the Wi-Fi MAC slots by the time of the first CCA after each LTE transmission; that this results both from $T_{\text {Idle }}$ being short and the Wi-Fi transmission times being of constant duration; and, as such, longer $T_{\text {Idle }}$ and/or more diversity in the Wi-Fi transmission times will cause $\rho_{L T E}$ to tend towards the Steady-state model value.

\section{CONCLUSION}

Two models were presented to estimate the LTE spectrum share under the frame-based LBT channel-access mechanism for Wi-Fi/LTE coexistence in the ETSI Standard. The first model was based on the assumption that the Wi-Fi traffic has sufficient time to reach steady-state between each clear channel assessment (CCA), whereas the second model included the dynamics of the Wi-Fi state distribution between CCAs.

The Dynamic model revealed that the LTE spectrum share, as a function of the Idle period, has a damped-oscillatory pattern that oscillates about the LTE spectrum share given by the Steady-state model. For Idle periods exceeding the Wi-Fi transmission times by approximately an order of magnitude, the oscillations subside and the Steady-state and Dynamic models converge.

A simple formula was offered that approximates the periodicity and peak locations of the damped-oscillatory pattern, enabling a simple method to approximately maximise the LTE spectrum share in which the Idle period is set to the first (and highest) peak of the damped-oscillatory pattern. A means of controlling the LTE spectrum share at a particular level, rather than just maximising the LTE spectrum share, was also offered.

A maximum LTE spectrum time share was found to be $32 \%$, for coexistence with networks comprising saturated WiFi stations operating under the current Wi-Fi technology, i.e. $802.11 \mathrm{n}(20 \mathrm{MHz})$. Moreover, a maximum LTE spectrum share was found to be $46 \%$, under the coexistence with advanced Wi-Fi technology, e.g., IEEE 802.11ac(160MHz).

\section{REFERENCES}

[1] IEEE std 802.11-2007, "Part 11: Wireless LAN Medium Access Control (MAC) and Physical Layer (PHY) specification," IEEE Std 802.11-2007, IEEE, Jun. 2007.

[2] IEEE std 802.11ac-2013, "Part 11: Wireless LAN Medium Access Control (MAC) and Physical Layer (PHY) Specifications, Amendment 4: Enhancements for Very High Throughput for Operation in Bands below 6 GHz," IEEE Std 802.11ac-2013, IEEE, 2013.

[3] M. Beluri, E. Bala, Y. Dai, R. Di Girolamo, M. Freda, J.-L. Gauvreau, S. Laughlin, D. Purkayastha, and A. Touag, "Mechanisms for LTE Coexistence in TV White Space," Dynamic Spectrum Access Networks (DYSPAN), 2012 IEEE International Symposium on, IEEE, pp.317-326, Oct. 2012.

[4] F. Liu, E. Bala, E. Erkip, and R. Yang, "A Framework for Femtocells to Access Both Licensed and Unlicensed Bands," Proceedings of the Third International Workshop on Indoor and Outdoor Femto Cells (IOFC), Princeton, NJ, USA, May 2011.
[5] C. Chen, R. Ratasuk, A. Ghosh, "Downlink Performance Analysis of LTE and WiFi Coexistence in Unlicensed Bands with a Simple Listen-beforetalk Scheme," in IEEE VTC-Spring, May 2015.

[6] P. Xia, Z. Teng, and J. Wu, "How Loud to Talk and How Hard to ListenBefore-Talk in Unlicensed LTE," in IEEE ICC Workshop on LTE in Unlicensed Bands: Potentials and Challenges, Jun. 2015.

[7] J. Jeon, H. Niu, Q. Li, A. Papathanassiou, G. Wu, "LTE with ListenBefore-Talk in Unlicensed Spectrum," in IEEE ICC Workshop on LTE in Unlicensed Bands: Potentials and Challenges, Jun. 2015.

[8] B. Jia and M. Tao, "A Channel Sensing Based Design for LTE in Unlicensed Bands," in IEEE ICC Workshop on LTE in Unlicensed Bands: Potentials and Challenges, Jun. 2015.

[9] A. Mukherjee, et al, "System Architecture and Coexistence Evaluation of Licensed-Assisted Access LTE with IEEE 802.11," in IEEE ICC Workshop on LTE in Unlicensed Bands: Potentials and Challenges, Jun. 2015.

[10] ETSI EN 301 893, Harmonized European Standard, "Broadband Radio Access Networks (BRAN); $5 \mathrm{GHz}$ high performance RLAN", v1.8.1, Mar. 2015.

[11] S. Han, Y.C. Liang, Q. Chen, and B.H. Soong, "Licensed-assisted access for LTE in unlicensed spectrum: A MAC protocol design," IEEE Journal on Selected Areas in Communications, 34(10), pp.2550-2561, 2016.

[12] R. Yin, G. Yu, A. Maaref, and G.Y. Li, "LBT-based adaptive channel access for LTE-U systems," IEEE Transactions on Wireless Communications, 15(10), pp.6585-6597, 2016.

[13] Y. Song, K.W. Sung, and Y. Han, "Coexistence of Wi-Fi and cellular with listen-before-talk in unlicensed spectrum," IEEE Communications Letters, 20(1), pp.161-164, 2016.

[14] F. Hao, C. Yongyu, H. Li, J. Zhang, and W. Quan, "Contention window size adaptation algorithm for LAA-LTE in unlicensed band," in Wireless Communication Systems (ISWCS), 2016 International Symposium on, pp. 476-480, IEEE, Sep., 2016.

[15] Y. Gao, X. Chu, and J. Zhang, "Performance Analysis of LAA and WiFi Coexistence in Unlicensed Spectrum Based on Markov Chain," in Global Communications Conference (GLOBECOM), 2016 IEEE, pp. 1-6, IEEE, Dec., 2016.

[16] Y. Kim, Y. Song, Y. Choi, and Y. Han, "Nonsaturated Throughput Analysis of Coexistence of Wi-Fi and Cellular With Listen-Before-Talk in Unlicensed Spectrum," IEEE Trans. Veh. Tech., vol. 66, no. 12, pp. 11425-11429, 2017.

[17] Y. Ma, D. G. Kuester, J. Coder, and W. Young, "Coexistence analysis of LTE and WLAN systems with heterogenous backoff slot durations," in IEEE Intl. Conf. on Commun. (ICC) 2017, pp. 1-7, IEEE, May 2017

[18] H. Lee, H. Kim, H. J. Yang, J. T. Kim, and S. K. Baek, "Performance Analysis of License Assisted Access LTE with Asymmetric Hidden Terminals," accepted to IEEE Trans. Mobile Comput., 2018.

[19] Y. Li, F. Baccelli, J.G. Andrews, T.D. Novlan, and J.C. Zhang, "Modeling and analyzing the coexistence of Wi-Fi and LTE in unlicensed spectrum," IEEE Transactions on Wireless Communications, 15(9), pp.63106326, 2016

[20] J. Jeon, Q. C. Li, H. Niu, A. Papathanassiou, and G. Wu, "LTE in the unlicensed spectrum: A novel coexistence analysis with WLAN systems," in GLOBECOM, IEEE, pp. 3459-3464, Dec. 2014.

[21] F. Capozzi, G. Piro, L. A. Grieco, G. Boggia, and P. Camarda, "Downlink packet scheduling in LTE cellular networks: Key design issues and a survey," IEEE Communications Surveys \& Tutorials, 15(2), pp.678-700, 2013.

[22] R. Love, R. Kuchibhotla, A. Ghosh, R. Ratasuk, B. Classon, and Y. Blankenship, "Downlink Control Channel Design for 3GPP LTE," in Proc. IEEE Wireless Communications and Networking Conference (WCNC), Las Vegas, Nevada, USA, pp.813-818, Apr. 2008.

[23] H. Wu, Y. Peng, K. Long, J. Ma, "Performance of Reliable Transport Protocol over IEEE 802.11 Wireless LAN: Analysis and Enhancement," in Proc. IEEE INFOCOM 2002, vol.2, pp.599-607, 2002.

[24] G. Bianchi, "Performance analysis of IEEE 802.11 distributed coordination funcitons," IEEE J. Sel. Areas Commun., vol.18, no.3, pp.535-547, Mar. 2000.

[25] R Core Team (2015). R: A language and environment for statistical computing. R Foundation for Statistical Computing, Vienna, Austria. URL https://www.R-project.org/.

[26] 3GPP TR 36.814 V9.2.0, "3rd Generation Partnership Project; Technical Specification Group Radio Access Network; Evolved Universal Terrestrial Radio Access (E-UTRA); Further advancements for E-UTRA physical layer aspects (Release 9)”, Mar. 2017. 


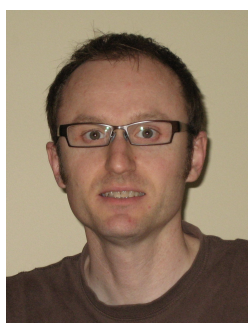

Gordon J. Sutton received a B.Sc. degree in Mathematics in 1993, B.E. degree in Systems engineering in 1995 and $\mathrm{PhD}$ in Control Theory in 1999, all from The Australian National University. He subsequently worked at ADC Australia designing optic fibre connectors and then at the Time Series Analysis Section of the Australian Bureau of Statistics. In 2006, Dr Sutton joined the Australian CSIRO as a statistician in the Quantitative Risk Management Stream of CSIRO Mathematics, Informatics and Statistics. In 2011, he joined the School of Chemistry, University of New South Wales, working in Bayesian statistics and chemometrics. Since 2015, Dr Sutton has been at the Global Big Data Technologies Centre, University of Technology Sydney, working on modelling Wi-Fi/LTE coexistence protocols. His interests include communications protocol modelling, WLAN, IoT, VANET, LTE, 5G, Markov processes, process analysis and control, forecasting, signal processing, particle filters, state space modelling and Bayesian statistics.

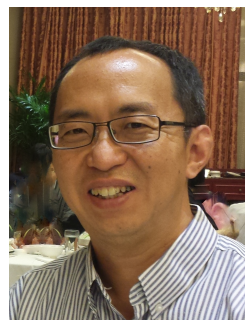

Ren Ping Liu (M'09-SM'14) is a Professor at the School of Electrical and Data Engineering in University of Technology Sydney, where he leads the Network Security Lab in the Global Big Data Technologies Centre. He is also the Research Program Leader of the Digital Agrifood Technologies in Food Agility CRC, a government/research/industry initiative to empower Australia's food industry through digital transformation. Prior to that he was a Principal Scientist at CSIRO, where he led wireless networking research activities. He specialises in protocol design and modelling, and has delivered networking solutions to a number of government agencies and industry customers. Professor Liu was the winner of Australian Engineering Innovation Award and CSIRO Chairman medal. His research interests include Markov analysis and QoS scheduling in WLAN, VANET, IoT, LTE, 5G, SDN, and network security. Professor Liu has over 100 research publications, and has supervised over $30 \mathrm{PhD}$ students.

Professor Liu is the founding chair of IEEE NSW VTS Chapter and a Senior Member of IEEE. He served as Technical Program Committee chair and Organising Committee chair in a number of IEEE Conferences. Ren Ping Liu received his B.E.(Hon) and M.E. degrees from Beijing University of Posts and Telecommunications, China, and the Ph.D. degree from the University of Newcastle, Australia.

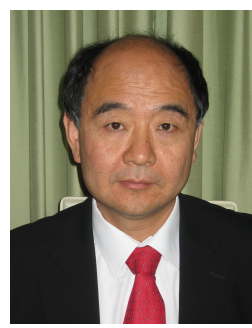

Y. Jay Guo (F'14) received a Bachelor Degree and a Master Degree from Xidian University in 1982 and 1984, respectively, and a $\mathrm{PhD}$ Degree from Xian Jiaotong University in 1987, all in China. He is a Fellow of the Australian Academy of Engineering and Technology, a Fellow of IEEE and a Fellow of IET, and a member of the College of Experts of Australian Research Council (ARC). He has won a number of most prestigious Australian national awards, and was named one of the most influential engineers in Australia in 2014 and 2015. His research interest includes antennas, $\mathrm{mm}$-wave and $\mathrm{THz}$ communications and sensing systems as well as big data. He has published over 300 research papers and holds 22 patents in antennas and wireless systems.

Prof Guo is the founding Director of Australia China Research Innovation Centre in Information and Electronics Technologies. He is a Distinguished Professor and the founding Director of Global Big Data Technologies Centre at the University of Technology Sydney (UTS), Australia. Prior to this appointment in 2014, he served as a Director in CSIRO for over nine years, directing a number of ICT research portfolios. Before joining CSIRO, he held various senior leadership positions in Fujitsu, Siemens and NEC in the U.K.

Prof Guo has chaired numerous international conferences. He is the International Advisory Committee Chair of IEEE VTC2017, General Chair of ISAP2015, iWAT2014 and WPMC'2014, and TPC Chair of 2010 IEEE WCNC, and 2012 and 2007 IEEE ISCIT. Prof Guo served as Guest Editor of special issues on Antennas for Satellite Communications and Antennas and Propagation Aspects of 60-90G Gz Wireless Communications, both in IEEE Transactions on Antennas and Propagation, Special Issue on Communications Challenges and Dynamics for Unmanned Autonomous Vehicles, IEEE Journal on Selected Areas in Communications (JSAC), and Special Issue on $5 G$ for Mission Critical Machine Communications, IEEE Network Magazine. 